\title{
Briófitas em unidades de conservação: Uma análise cienciométrica
}

\author{
Bryophytes in conservation units: A scientometric analysis \\ Briofitas en unidades de conservación: Un análisis cientométrico
}

Recebido: 08/06/2021 | Revisado: 16/06/2021 | Aceito: 21/06/2021 | Publicado: 04/07/2021

\author{
Regigláucia Rodrigues de Oliveira \\ ORCID: https://orcid.org/0000-0003-3916-3686 \\ Universidade Federal do Piauí, Brasil \\ E-mail: regiglaucia@hotmail.com \\ Ivanilza Moreira de Andrade \\ ORCID: https://orcid.org/0000-0001-6059-8540 \\ Universidade Federal do Delta do Parnaíba, Brasil \\ E-mail: ivanilzaandrade@hotmal.com
}

\begin{abstract}
Resumo
Unidades de Conservação são espaços instituídos pelo poder público com finalidade de conservar características naturais relevantes. As Briófitas, além de constituir a flora dessas áreas, são mais vulneráveis à perda de ambientes do que as demais plantas terrestres, alia-se a isto o fato de não existir qualquer coleção de material vivo, e só podem ser conservadas pela preservação de áreas naturais. Objetivou levantar dados sobre Briófitas em UCs visando ampliar o conhecimento do grupo em áreas protegidas, e ainda, detectar as possíveis carências de estudos em diversas áreas do Brasil. Na base de dados Web of Science ${ }^{\mathrm{TM}}$ foi feita uma busca utilizando descritores, combinados com operador booleano AND dispostos da seguinte maneira: byophytes AND protected area, bryophythes AND unit conservation, bryophytes AND nacional park, bryophytes AND state park. Foram registrados 119 artigos publicados para diversos países entre os anos de 2010 a 2020. Uma elevada quantidade concentra-se nos anos de 2014 e 2016. A Inglaterra é o país que mais publica sobre o tema, e o Brasil ocupa segunda posição. A maioria dos resultados são concentrado em Ciência Vegetal (35\%), e Ciência Ambiental (28\%), justificado pelo fato de serem áreas mais relacionadas com o tema abordado. Esse estudo aponta algumas lacunas sobre o conhecimento de briófitas em UCs, ressaltando a necessidade de pesquisas com esses vegetais em algumas regiões do Brasil, onde vários estados do Nordeste continuam subamostrados em relação à brioflora. E reforça, a importância de escolher periódicos indexados em relevantes bases de dados, para maior visualização das pesquisas.
\end{abstract}

Palavras-chave: Áreas protegidas; Antóceros; Musgos; Hepáticas.

\begin{abstract}
Conservation Units (CUs) are territorial spaces instituted by the government that have the purpose of conserving relevant natural characteristics. The Bryophytes, which in addition to constituting the flora of these areas, are more vulnerable to loss of environments than other terrestrial plants, in addition to this the fact that there is no collection of living material, and thus, can only be conserved by the preservation of natural areas. The objective was collect data on work with Bryophytes in CUs to complete the group's knowledge in protected areas, and also to detect possible lack of studies in different areas of Brazil. In the Web of Science TM database, a search was performed using the descriptors, combined with the boolean operator AND arranged as follows: bryophytes AND protected area, bryophytes AND unit conservation, bryophytes AND national park, bryophytes AND state park. There were 119 articles published for different countries between the years 2010 to 2020. A large number of articles were concentrated in the years 2014 and 2016. England is the country that publishes the most on the topic and Brazil occupies second place. The search obtained the majority of the results concentrated in the Plant Science (35\%), and Environmental Science (28\%), among these, the areas most related to the topic addressed. This study draws attention to some gaps regarding the knowledge of bryophytes in CUs, highlighting the need for research with this plant group in some regions of Brazil, where several states in the Northeastern continue to be undersampled in relation to bryophyte. And reinforce the importance of choosing indexed journals in relevant databases, for greater visualization of researches.
\end{abstract}

Keywords: Protected area; Hornworts; Mosses; Liverworts.

\section{Resumen}

Unidades de Conservación (UC) son espacios territoriales establecidos por el gobierno que tienen el propósito de conservar características naturales relevantes presentes en el área. Las briofitas, que además de constituir la flora de estas áreas, son más vulnerables a la pérdida de ambientes que otras plantas terrestres, a esto se suma el hecho de que no existe recolección de material vivo, por lo tanto, solo se puede conservar mediante la preservación de áreas naturales. El objetivo fue recolectar datos sobre el trabajo con briófitas en UCs. Completar el conocimiento del grupo 
en áreas protegidas, y también detectar posible falta de estudios en diferentes áreas de Brasil. En la base de datos Web of Science TM, se realizó una búsqueda usando los descriptores, combinados con el operador booleano y organizado de la siguiente manera: briofitas $\mathrm{Y}$ area protegida, briofitas $\mathrm{Y}$ conservación de unidades, briofitas $\mathrm{Y}$ parque nacional, briofitas Y parque estatal. Se publicaron 119 artículos para diferentes países entre los años 2010 a 2020 . Una gran cantidad de artículos se concentraron en los años 2014 y 2016. Inglaterra es el país que más publica sobre el tema y Brasil ocupa el segundo lugar. La búsqueda obtuvo la mayoría de los resultados concentrados en Ciencias Vegetales (35\%), e Ciencias Ambientales (28\%), justificado por el hecho de que son, entre estos, los ámbitos más relacionados con el tema abordado. Este estudio llama la atención sobre algunas lagunas con respecto al conocimiento de las briofitas en UC, destacando la necesidad de investigar con estas plantas en algunas regiones de Brasil, dónde varios estados del noreste continúan siendo submuestreados en relación a las briofitas. E refuerzan la importancia de elegir revistas indexadas en bases de datos relevantes, para una mayor visualización de las investigaciones.

Palabras clave: Áreas protegidas; Antoceros; Hepáticas; Musgos.

\section{Introdução}

Devido ao crescente número de impactos ambientais e ameaças à diversidade biológica, deu-se a necessidade de criação de áreas naturais, visando a proteção da biodiversidade. A primeira área protegida, com fins de resguardar a natureza, cujo uso seria controlado pelo poder público, elementos semelhantes às atuais Unidades de Conservação, é datada do século XIX, com a criação do Parque Nacional de Yellowstone, nos Estados Unidos em 1872, com objetivo de preservar suas paisagens “intocáveis" para as gerações futuras (WWF-Brasil, 2010). Com a disseminação da ideia houve algumas tentativas da criação de outras áreas protegidas, algumas sem sucesso. Porém, seguindo essa iniciativa, foi criada a primeira Unidade de Conservação (UC) brasileira em 1937, na divisa dos estados de Minas Gerais e Rio de Janeiro, reconhecido por Parque Nacional de Itatiaia (Medeiros, 2014).

As Unidades de Conservação são espaços territoriais instituídos pelo poder público que tem a finalidade de conservar características naturais relevantes presentes na área, com a função de assegurar a representatividade de amostras significativas e ecologicamente viáveis das diferentes populações, habitats e ecossistemas do território nacional e das águas jurisdicionais, preservando o patrimônio biológico existente (MMA, 2019; WWF Brasil, 2020). Além disto, apresentam objetivos de conservação e limites definidos, sob regime especial de administração, ao qual se aplicam garantias adequadas de proteção. Esses espaços territoriais, especialmente protegidos, são mundialmente reconhecidos como instrumentos fundamentais para conservação in situ de espécies, populações, comunidades e ecossistemas (Rylands \& Brandon, 2005).

A União Internacional para a Conservação da Natureza (IUCN) foi criada em 1948 com o intuito de influenciar, encorajar e auxiliar sociedades em todo o mundo a criar áreas protegidas que conservem a integridade e diversidade da natureza, além de assegurar que toda utilização de recursos naturais seja feita de modo equitativo e ecologicamente sustentável. A IUCN apresenta o conceito de área protegida como sendo "uma área de terra/mar especialmente dedicada à proteção e manutenção da biodiversidade e de seus recursos naturais e culturais associados, e manejados através de instrumentos legais e outros efetivos" (IUCN, 2020).

No Brasil, as Unidades de Conservação são regidas pela Lei 9.985/2000 que criou o Sistema Nacional de Unidades de Conservação da Natureza (SNUC) e estabelece critérios e normas para a criação, implantação e gestão das UCs. O SNUC é composto pelo conjunto de Unidades de Conservação federais, estaduais, municipais e particulares, distribuídas em doze categorias de manejo (MMA, 2019).

As UCs estão organizadas em dois grupos: O primeiro é composto por Unidades de Proteção Integral que tem como finalidade preservar a natureza, sendo admitido apenas o uso indireto dos recursos naturais, e por isso as regras e normas são restritivas, com as seguintes categorias: Estação Ecológica, Reserva Biológica, Parque Nacional, Refúgio de Vida Silvestre e Monumento Natural. O segundo grupo são as Unidades de Uso Sustentável, que concilia a conservação da natureza com o uso sustentável de parte dos recursos naturais. Esse grupo é constituído pelas categorias: Área de Proteção Ambiental, Área de 
Relevante Interesse Ecológico, Floresta Nacional, Reserva Extrativista, Reserva de Fauna, Reserva de Desenvolvimento Sustentável e Reserva Particular do Patrimônio Natural (MMA, 2020).

Com a criação das Unidades de Conservação, o patrimônio natural e ambiental do Brasil tem sido protegido desde 1937. Desde então, as áreas abrangidas por UC têm aumentado, especialmente nos últimos anos. Vale lembrar que algumas UCs brasileiras são reconhecidas pela UNESCO como sítios do Patrimônio Mundial Natural por possuírem importância tão extraordinária ao ponto de serem consideradas como patrimônio da humanidade para as presentes e futuras gerações (MMA, 2011).

Áreas protegidas são criadas para garantir a sobrevivência de todas as espécies, e também para proteger locais de grande beleza cênica, como montanhas, serras, cachoeiras, cânions, rios ou lagos. Além disto, contribuir para regular o clima, abastecer os mananciais de água e proporcionar qualidade de vida às populações humanas (IUCN, 2019). Nesse contexto, cabe mencionar as Briófitas que além de compor a flora dessas áreas, também são consideradas eficientes bioindicadores da qualidade ambiental (Santos, 2003).

Ademais, as briófitas geralmente são quase totalmente dependentes da vegetação formada por outros grupos de plantas para sua sobrevivência, a destruição de matas e outros tipos de vegetação natural tem o efeito de eliminar quase completamente as espécies de briófitas associadas (Sherped, 2003; Reis, 2018). Neste sentido, briófitas provavelmente são mais vulneráveis à perda de ambientes do que as angiospermas e não existe qualquer coleção de material vivo ou armazenamento de esporos. Em geral, não podem ser facilmente cultivadas e assim, só podem ser conservadas pela preservação da vegetação natural (Sherped, 2003).

As briófitas compreendem três divisões, representadas pelas hepáticas (Marchantiophyta) antóceros (Anthocerotophyta) e musgos (Bryophyta), plantas que compartilham entre si dominância da fase haplóide na forma de gametófito e a fase esporofítica diplóide nutricionalmente depedente do gametófito (Goffinet et al., 2009; Glime, 2020), ausência de um sistema vascular lignificado, absorvem os nutrientes e água em toda a superfície do gametófito, e dependência da água para a reprodução sexuada, e assim, ocorrem com maior frequência em ambientes úmidos e sombreados. São mais abundantes e diversos em florestas tropicais, mas podem ser encontradas em todos os tipos de ambientes, do polar a regiões áridas, incluindo desertos (Glime, 2020).

No mundo, estão representadas por cerca de 18.150 espécies (Goffinet et al., 2009), caracterizando-se como o segundo maior grupo de plantas terrestres (Frahm, 2003). Estima-se que 1/3 das espécies distribuídas pelo mundo está na região Neotropical (Gradstein et al., 2001) e segundo Costa \& Peralta (2015) o Brasil abriga 1.524 espécies da brioflora.

A distribuição espacial das briófitas pode ser moldada pelos filtros existentes numa área, a exemplo, filtros abióticos locais têm-se abertura do dossel, rochosidade, luminosidade e umidade, assim como outras variáveis geoclimáticas que também são importantes filtros ambientais (Honorio Conorado et al., 2009). A variedade de riqueza, diversidade e composição das espécies de briófitas, e suas formas de vida estão claramente ligadas a condições microclimáticas, mas especialmente a disponibilidade de luz e água (Mägdefrau, 1982). Pesquisas com briófitas em UCs têm sido desenvolvidas nos útimos anos.

Diante disso, objetivou levantar informações sobre trabalhos que abordem Briófitas em Unidades de Conservação visando ampliar o conhecimento da existência desse grupo vegetal- muitas vezes negligenciado, em áreas protegidas, e ainda, detectar as possíveis carências de estudos em diversas áreas do Brasil, resultados que servirão de subsídios para futuras pesquisas aplicadas ao conhecimento dessas plantas especialmente em áreas protegidas. 


\section{Metodologia}

O trabalho é caracterizado como cienciométrico, definido por Darosi e Anderle, (2014) como a área que trata da análise de aspectos quantitativos referentes à geração, propagação e utilização de informações científicas, com o fim de contribuir para o melhor entendimento do mecanismo de pesquisa científica como uma atividade social.

Foi utilizada a base Web of Science ${ }^{\mathrm{TM}}$ (Clarivate Analytics) que oferece acesso à coleção principal da base de dados, permitindo acesso a referências e resumos em todas as áreas do conhecimento. Por meio dessa base de dados, estão disponíveis ferramentas para análise de citações, referências, índice $\mathrm{h}$, permitindo análises bibliométricas. Atualmente, cobre aproximadamente 12.000 periódicos (CAPES, 2020).

Foi realizada uma busca com as combinações de palavras sinônimas que englobassem os termos de interesse do trabalho: Briófitas em Unidades de Conservação. A busca foi realizada em outubro de 2020, utilizando a rede da Universidade Federal do Piauí que disponibiliza o acesso ao portal de periódicos Capes que oferece acesso a diversas bases de dados. Para a prospecção científica, no campo de "busca" foram utilizados descritores, combinados com o operador booleano AND dispostos da seguinte maneira: byophytes AND protected area, bryophythes AND unit conservacion, bryophytes AND nacional park, bryophytes AND State park. Justifica-se o uso dos dois últimos descritores em função dessas categorias, Parques Nacionais e Estaduais estarem incluídos em UCs de proteção integral, áreas bastante relevantes no que tange a preservação.

A investigação foi delimitada em artigos publicados, entre os anos de 2010 a 2020, com o intuito de abranger a última década e, assim, descrever a evolução dos estudos com essa temática neste período. Muitas vezes a busca foi restringida, selecionando artigos de acesso aberto (Open Acess). Os resultados foram exportados para o Excel e quando necessário, transferidos para um arquivo doc.x para uma melhor utilização dos dados.

Os artigos foram analisados pelo resumo e, quando necessário, lidos na íntegra. Em seguida foram analisadas as seguintes variáveis: autor, periódico, título, palavras-chave, volume, número do periódico, ano, local, país.

Alguns artigos não puderam ser incluídos na análise, devido às informações do resumo não tratar da temática de interesse dos autores, quando estes eram vistos na íntegra, não foram encontradas informações suficientes para estes serem incluídos na análise.

\section{Resultados e Discussão}

Ao fazer a busca, utilizando os termos: Briófitas e Áreas Protegidas, combinados (Bryophytes AND Protected Area), obteve-se um total de 119 artigos, entretanto quando se refinou buscando por aqueles de acesso aberto, esse número caiu para 43. Foi verificada também, a quantidade de trabalhos publicados por ano na última década (Figura 1), observou-se que houve um maior número de publicações nos anos de 2014 e 2016 (18 e 16 artigos, consecutivamente). Pode-se inferir, que esse resultado reflete a preocupação diante da crescente onda de desastres ambientais, ocorridos até mesmo em áreas protegidas, observada nos últimos anos, no Brasil e no mundo. 
Figura 1. Número de artigos, com acesso aberto, publicados para diversos países (Brasil, Inglaterra, Suíça, Suécia, Bélgica, Canadá, Finlândia, Polônia, Portugal e Espanha) com Briófitas em Áreas Protegidas entre 2010 e 2020.

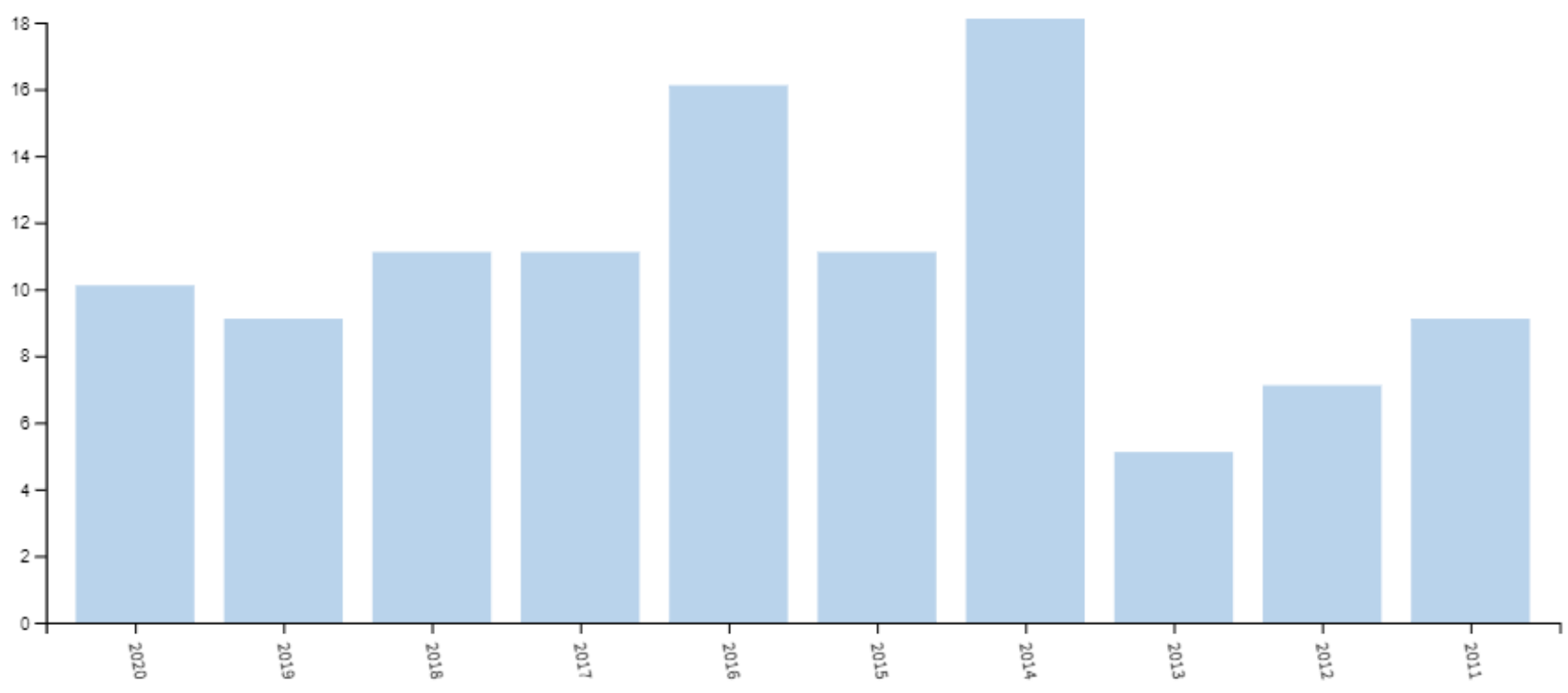

Fonte: Web of Science TM (2020).

Analisou-se ainda que, para o mundo na última década, houve um crescimento expressivo do número de citações de trabalhos com essa temática com destaque para o ano de 2019, acompanhado de uma recente queda no ano de 2020 (Figura 2).

Figura 2. Número de citações utilizando os termos: Briófitas em Áreas Protegidas entre 2010-2020, para os diversos países do mundo.

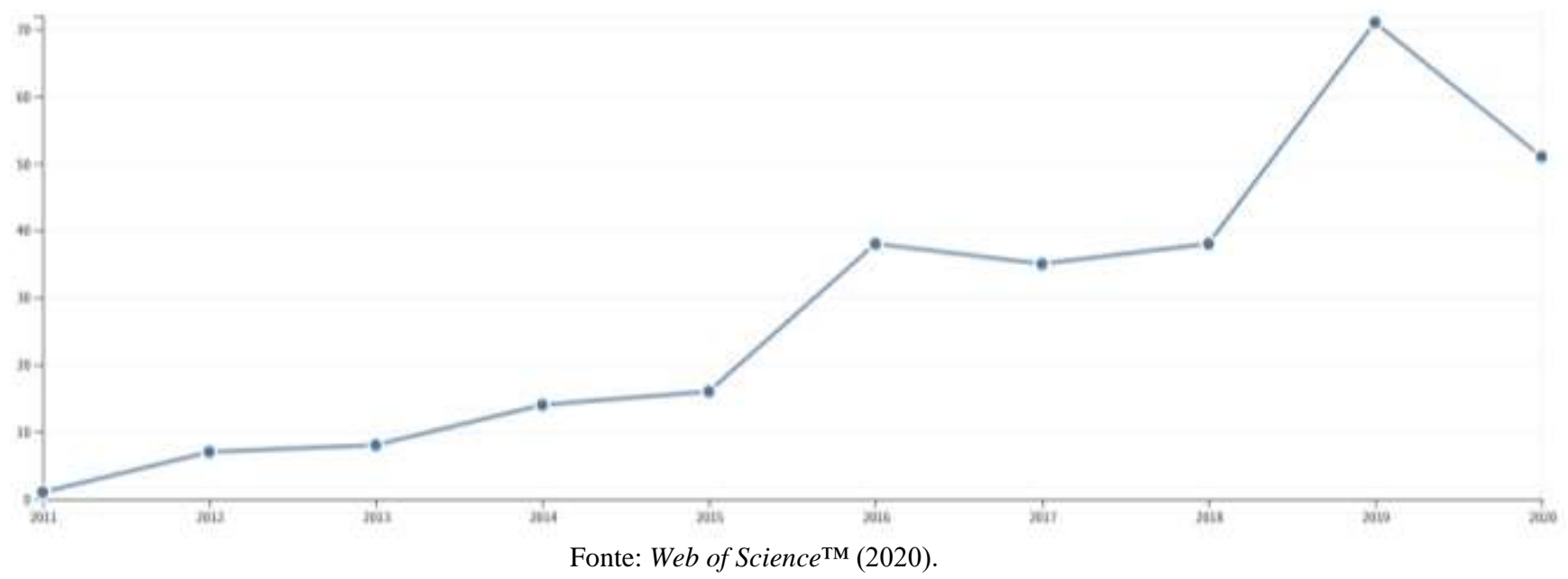

Refinando-se ainda mais a busca, selecionando a opção acesso aberto: (open access) e países/regiões, podemos analisar que o Brasil, juntamente com Suécia e Suíça ocupam segundo lugar no ranking de publicações com o tema Briófitas em Áreas Protegidas, ficando atrás, apenas de Inglaterra, em termos de publicações com a temática, levando em consideração os resultados apresentados pela base de periódicos do Web of Science ${ }^{\mathrm{TM}}$ (Figura 3). Segundo dados da Flora do Brasil (2020), o Brasil está representado atualmente por 1.575 espécies de briófitas, distribuídos em 15 espécies de antóceros, 670 hepáticas e 890 de musgos. Considera-se que talvez um quinto de todas as espécies no mundo ocorrem no país (Sherped, 2003). 
Neste contexto, cabe ainda mencionar uma possível justificativa para estes dados, uma vez que a Inglaterra ocupa o topo das publicações com o tema, vale ressaltar que este país possui uma expressiva preocupação sobre responsabilidade civil por danos ao ambiente natural, influenciado principalmente pela pressão exercida sobre o meio ambiente, e começou a ser alterado com maior intensidade, a partir da Revolução Industrial iniciada no século XVIII. E ainda, foi também na Inglaterra, já no século XIX, que surgiu a responsabilidade objetiva por danos ao meio ambiente, que teve grande repercussão em outros países do mundo (Delgado, 2008; Coelho \& Rezende, 2016).

Figura 3. Posição ocupada pelo Brasil, em relação aos outros países em número de publicações com Briófitas em Áreas Protegidas entre 2010-2020.

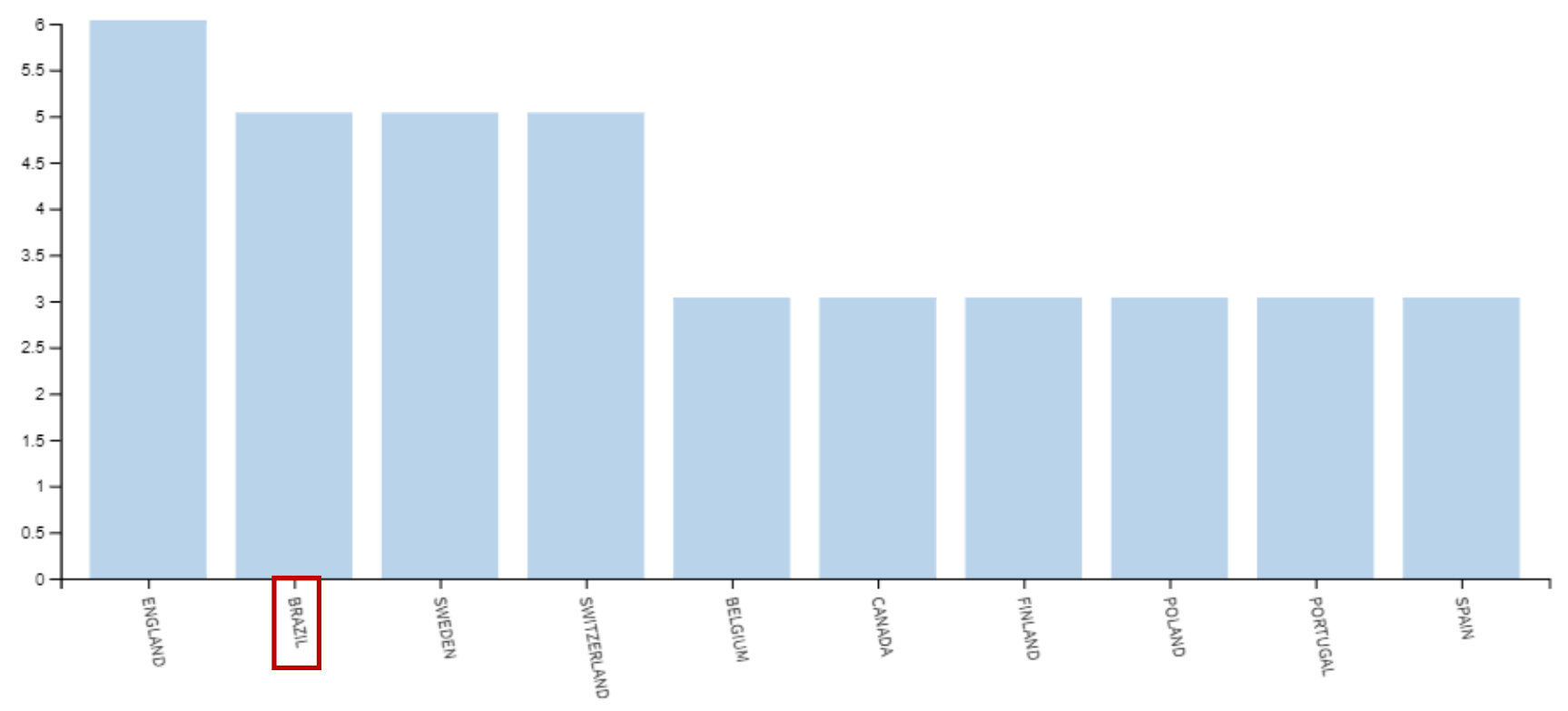

Fonte: Autores (2020).

Ao verificar as principais categorias nas quais os artigos foram publicados (Figura 4), constatou-se o maior número de trabalhos em Ciência Vegetal (40), seguido por Ciências Ambiental (33), Ecologia (32), Conservação da Biodiversidade (22) e Ciência Florestal (16), sendo estas, as áreas mais representativas em número de publicações com a temática abordada, segundo a base Web of Science (2020). As categorias: Multidisciplinar (6), Biologia, Geografia Física e Geociências com quatro publicações cada uma e Zoologia com três publicações. Agronomia, Ciências Atmosféricas e Meteorológicas, Saúde Pública Ambiental e Ocupacional, além de Recursos Aquáticos com duas cada. As demais áreas (Micologia, Microscopia, Biologia Evolutiva, Estudos Ambientais, Arqueologia, Política Econômica e Agricultura, Ciência Animal e Agropecuária), com apenas um artigo, juntas essas categorias totalizaram 109 artigos. Pode se observar que estes resultados refletem uma certa afinidade com a categoria de publicação e o tema pesquisado, mostrando assim, ainda uma forte tendência em relação à dissociação das áreas, diante disso, presume-se que ainda falta uma maior correlação entre as grandes áreas de estudo. 
Figura 4. Áreas de publicação dos artigos científicos publicados com Briófitas em Áreas Protegidas entre 2010 -2020.

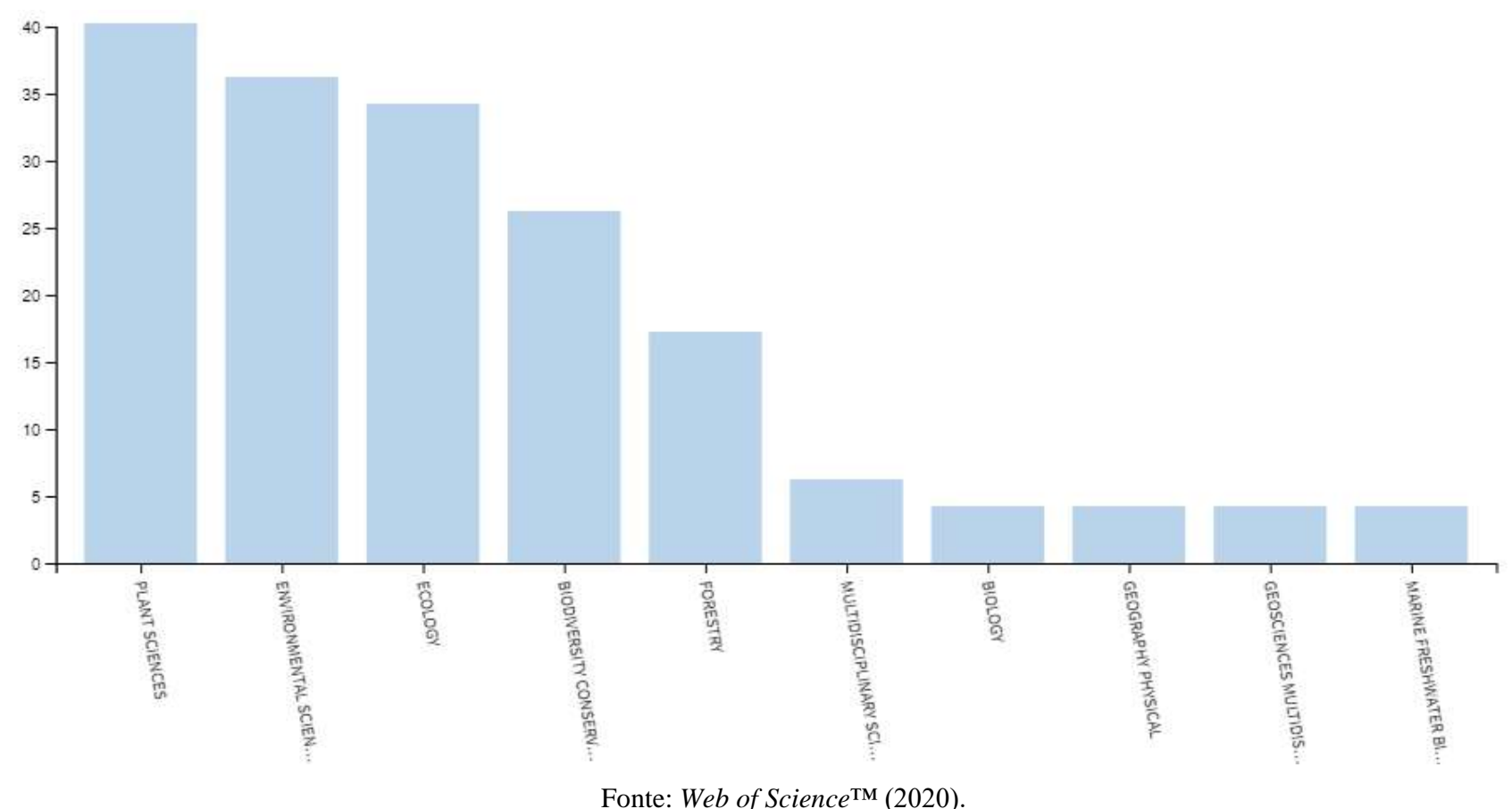

Dos 109 artigos localizados nessa base de dados para o mundo, restringindo a busca por países/regiões, selecionando a opção Brasil, obteve-se um total de 09 artigos (Tabela 1). Resultado semelhante foi encontrado ao usar os termos: Unit Conservacion AND bryophytes. Que resultou em 52 periódicos, sendo que desses 06 artigos referem-se ao Brasil, observou-se nessa busca, uma grande sobreposição dos artigos encontrados, pelo fato de utilizar termos sinônimos.

Entretanto, ao analisar um dos resumos, foi observado que um deles não se encaixava na busca, uma vez que tratava de um estudo desenvolvido apenas com duas espécies de musgos do gênero Sphagum em turfeiras holoárticas. Após análise do texto na íntegra, foi verificado que esse resultado, se deu em função do termo Unit Conservacion, que ao longo do artigo foi mencionado o grupo de pesquisa: Plant Ecology and Nature Conservation Group, fazendo com que este periódico aparecesse na busca. Optou-se, como critério nessa busca, não restringir os termos usando aspas, uma vez que ao fazer o uso de " " não foram localizados na base resultados com essa combinação de termos. 
Tabela 1. Artigos localizados em busca realizada na base de dados Web of Science ${ }^{\mathrm{TM}}$, publicados para o Brasil entre os anos de 2010-2020.

\begin{tabular}{|c|c|c|c|}
\hline Autores & Título do Artigo & Periódico & $\begin{array}{c}\text { Ano de } \\
\text { Publicação }\end{array}$ \\
\hline $\begin{array}{l}\text { Pereira, MR; Ledent, A; } \\
\text { Mardulyn, P; Zartman, CE; } \\
\text { Vanderpoorten, A }\end{array}$ & $\begin{array}{l}\text { Maintenance of genetic and morphological } \\
\text { identity in two sibling Syrrhopodon species } \\
\text { (Calymperaceae, Bryopsida) despite } \\
\text { extensive introgression }\end{array}$ & $\begin{array}{l}\text { Journal of Systematics and } \\
\text { Evolution }\end{array}$ & 2019 \\
\hline $\begin{array}{l}\text { Kaffer, MI; Koch, NM; } \\
\text { Martins, SMD; Vargas, } \\
\text { VMF }\end{array}$ & $\begin{array}{l}\text { Lichen community versus host tree bark } \\
\text { texture in an urban environment in southern } \\
\text { Brazil }\end{array}$ & Iheringia Serie Botanica & 2016 \\
\hline $\begin{array}{l}\text { Fagundes, DN; Tavares- } \\
\text { Martins, ACC; Ilkiu-Borges, } \\
\text { AL; Moraes, EDR; dos } \\
\text { Santos, RDP }\end{array}$ & $\begin{array}{l}\text { Richness and ecological aspects of } \\
\text { bryophyte communities (Bryophyta and } \\
\text { Marchantiophyta) of a fragment of Terra } \\
\text { Firme Forests in Gunma Ecological Park, } \\
\text { Municipality of Santa Barbara, Para, Brazil }\end{array}$ & Iheringia Serie Botanica & 2016 \\
\hline Silva, MPP; Porto, KC & $\begin{array}{l}\text { Diversity of bryophytes in priority areas for } \\
\text { conservation in the Atlantic forest of } \\
\text { northeast Brazil }\end{array}$ & Acta Botanica Brasilica & 2015 \\
\hline $\begin{array}{l}\text { Deheuvels, O; Rousseau, } \\
\text { GX; Quiroga, GS; Franco, } \\
\text { MD; Cerda, R; Mendoza, } \\
\text { SJV; Somarriba, E }\end{array}$ & $\begin{array}{l}\text { Biodiversity is affected by changes in } \\
\text { management intensity of cocoa-based } \\
\text { agroforests }\end{array}$ & Agroforestry Systems & 2014 \\
\hline $\begin{array}{l}\text { Macedo, LPC; Ilkiu-Borges, } \\
\text { AL }\end{array}$ & $\begin{array}{l}\text { Richness of Marchantiophyta and Bryophyta } \\
\text { in a protected area of the Brazilian Amazon }\end{array}$ & Acta Botanica Brasilica & 2014 \\
\hline Camara, PEAS; van Rooy, J & $\begin{array}{l}\text { A new species of Sematophyllum } \\
\text { (Sematophyllaceae) from South Africa and a } \\
\text { key to the southern African species of the } \\
\text { genus }\end{array}$ & Bryologist & 2014 \\
\hline $\begin{array}{l}\text { Silva, MPP; Kamino, LHY; } \\
\text { Porto, KC }\end{array}$ & $\begin{array}{l}\text { Is the current network system of protected } \\
\text { areas in the Atlantic Forest effective in } \\
\text { conserving key species of bryophytes? }\end{array}$ & $\begin{array}{l}\text { Tropical Conservation } \\
\text { Science }\end{array}$ & 2014 \\
\hline $\begin{array}{l}\text { Barros, FSM; de Siqueira, } \\
\text { MF; da Costa, DP }\end{array}$ & $\begin{array}{l}\text { Modeling the potential geographic } \\
\text { distribution of five species of Metzgeria } \\
\text { Raddi in Brazil, aiming at their conservation }\end{array}$ & Bryologist & 2012 \\
\hline
\end{tabular}

Fonte: Dados coletados na pesquisa.

Além deste, três outros artigos localizados nessa busca, não se encaixavam com os objetivos da pesquisa. Um deles, realizado com Líquens em área urbana no Sul do Brasil (Kaffer et al, 2016). O trabalho não aborda briófitas, nem Área Protegida, entretanto, o fato de ter sido localizado na busca, deu-se em decorrência do termo Protected area, utilizado sem " " nos termos da busca, fazendo com que a base de dados mostrasse esse artigo como possibilidade.

O segundo artigo a ser eliminado por não se encaixar nos objetivos da pesquisa, foi localizado por mencionar trabalhos realizados no Brasil (por meio da restrição: regiões/países), e se trata de uma pesquisa desenvolvida em sistemas agroflorestais com plantação de cacau na Bahia. Objetivando fazer análise das diferenças na composição e estrutura da vegetação de 36 agroflorestas de cacau, que podem afetar a diversidade de pequenos mamíferos, anfíbios, répteis, solo e serapilheira macroinvertebrados, epífitas encontrados em árvores de cacau e plantas associadas que fornecem habitats para numerosas espécies dependentes da floresta de alto valor de conservação. Embora o artigo tenha sido mostrado como possibilidade sobre o assunto da busca (devido aos termos conservation e mosses que foram encontrados no texto na 
íntegra), justifica-se pelo fato dos autores mencionarem, nos resultados espécies de musgos crescendo nos caules das plantas de cacau (Deheuvels et al. 2014), embora o estudo não tenha sido desenvolvido em Unidades de Conservação.

O terceiro artigo eliminado por não se enquadrar na busca, diz respeito a citação de uma nova espécie de musgo, Sematophyllum (Sematophyllaceae) da África do Sul e uma chave de identificação para as espécies do gênero na África Austral, esse artigo foi localizado, por mencionar que esta espécie de briófita é bem distribuída e não está particularmente ameaçada, na medida em que ocorre em várias áreas protegidas (Camara \& Van Rooy, 2014), um dos termos utilizado na busca.

Ao fazer análise dos artigos selecionados que se enquadravam na busca, constatou-se que três deles contemplam a região Norte do país. Mais especificamente, coletas de briófitas (hepáticas e musgos) realizadas em importantes áreas protegidas da Amazônia. Seguida pela região Nordeste, também com três artigos, sendo que dois deles são para o estado do Pernambuco, amostrando briófitas do domínio fitogeográfico Floresta Attântica (Silva \& Pôrto, 2015, Pôrto et al., 2014), e outro para a Bahia (Deheuvels et al., 2014).

Nos resultados do trabalho de Costa et al., (2012), utilizando o gênero de hepática/Metzgeria (Marchantiophyta) visando apoiar a conservação de espécies de briófitas no Brasil, os autores destacaram a importância do desenvolvimento de estratégias de conservação e planos de ação para evidenciar o grande potencial dessas espécies, além de identificar lacunas de conhecimento sobre essas plantas, além de fornecer ferramentas para examinar o status de conservação das espécies. Esta pesquisa foi realizada com base em espécies coletadas para vários estados brasileiros e que estão depositados no Herbário do Jardim Botânico do Rio de Janeiro/ Herbário RB. Vale mencionar que nem todas as coletas foram oriundas de Unidades de Conservação, entretanto, objetivou-se com este estudo, propor o uso de métodos de modelagem de nicho ecológico para estimar a extensão da ocorrência potencial de espécies. Ademais, os autores destacam que compreender a distribuição espacial das espécies é essencial para a conservação da biodiversidade, e o uso de modelos de distribuição potencial na análise biogeográfica é uma ferramenta importante para a conservação de espécies raras ou ameaçadas de extinção (Costa et al., 2012).

Embora tenha conhecimento da existência de outros artigos publicados abordando briófitas em Unidades de Conservação, para diversas regiões do Brasil, muitos deles não foram localizados nessa busca. E com isso, cogitar possibilidades para esta não localização de alguns artigos. Supõe-se que os artigos foram publicados em revistas científicas que não se encontram indexadas, fazendo com que estes, não tenham sido encontrados nessa busca.

Então, visando ampliar o número de artigos localizados, optou-se por tentar novas combinações de termos, sendo mais específicos em relação às categorias das UCs. Quando se fez a busca combinada com os termos Bryophytes AND National Park, obtendo os seguintes resultados: 28 artigos, desses, 12 eram com acesso aberto, que foram analisados, visando verificar a distribuição nos diferentes estados e domínios fitogeográficos brasileiros.

Constatou-se, que a maioria dos trabalhos publicados com briófitas em Parques Nacionais no Brasil foram realizados na região Sudeste do país, contemplando o domínio fitogeográfico Floresta Atlântica, seguido pela região Centro-Oeste, com destaque para o estado de Minas Gerais, abrangendo PARNA do Cangas e o domínio fitogeográfico do Cerrado. Nessa busca, teve-se ainda resultados do trabalho realizado no Parque Estadual Serra do Mar/SP. O bioma Amazônia também foi contemplado, com pesquisas realizadas nos estados do Amapá, Amazonas, Belém e Acre. Para Parques Nacionais, estudo com briófitas na região Nordeste foram representados pelo estado do Pernambuco, em áreas de fragmentos de Floresta Atlântica.

Quando se buscou por Parques Estaduais usando os termos Bryophytes AND State Park, houve sobreposição dos resultados. Muitos artigos apareceram novamente devido ao termo Park. Ao restringir a busca "State Park", houve uma queda de 26 para 10 artigos encontrados. Em meio aos artigos localizados nessa busca, observou-se um abordando Vespas (Hymenoptera/Vespidae) realizado no Parque Estadual de Ibitipoca, Minas Gerais, esse artigo com título: Nesting Behaviour 
of a Neotropical Social Wasp Mischocyttarus saussurei Zikán, 1949 (Hymenoptera, Vespidae), que à primeira vista parecia não se enquadrar na busca. Entretanto, ao analisar o artigo na íntegra, foi constatado que isso deu-se em função dos resultados deste artigo, mencionar que esses animais invertebrados utilizam espécies de briófitas para camuflagem, espécies de hepáticas e musgos (Milani et al, 2020).

Com a utilização de novos termos, percebeu-se que houve um aumento na quantidade de trabalhos localizados nessa busca, abordando outros estados e regiões do país. Porém, os resultados ainda não contemplam a realidade das publicações com briófitas em Unidades de Conservação, nem todos os Biomas brasileiros. Por exemplo, tem-se o conhecimento que os estados do Piauí e Maranhão, nos últimos anos, entraram para o cenário de trabalhos com Briófitas em UCs, resultados mostrados nos trabalhos desenvolvidos por Oliveira et al (2020), que registram 90 espécies distribuídas em 43 gêneros e 23 famílias, para o Parque Nacional das Sete Cidades, Piauí. Com esses resultados os autores destacaram a relevância dessa UC, bem como a continuidade de trabalhos que visem catalogar a brioflora dessas áreas, importantes do domínio fitogeográfico Caatinga.

Costa et al., (2015) registraram 14 espécies de musgos distribuídas em nove famílias e 10 gêneros, duas espécies de hepáticas com dois gêneros e duas famílias, na Área de Proteção Ambiental Buriti do Meio, no município de Caxias/Maranhão. Para o mesmo estado, Oliveira et al., (2018) catalogaram 38 espécies de musgos acrocárpicos representados por 18 gêneros e sete famílias, no Parque Nacional Chapada das Mesas em uma área ecotonal importante do Nordeste brasileiro.

Portanto, pode-se confirmar que as proposições levantadas anteriormente, a respeito da não localização de alguns artigos nessa busca, foram confirmadas, uma vez que ao longo da pesquisa foi confirmado que alguns artigos ficaram de fora da busca, mesmo com a diversidade de termos utilizados durante a pesquisa. Neste caso, pode-se concluir que estes apesar de serem publicados, não foram indexados na base de dados utilizadas nessa pesquisa. Aproveitando o ensejo, para ressaltar a importância do material que o pesquisador deseja divulgar, uma vez que, é fundamental fazer boas escolhas na hora da publicação dos dados da pesquisa, objetivando que esta tenha maior visibilidade e atinja maior número de pessoas, significa dizer que é indispensável que o material seja publicado em periódicos que possua alto impacto, e com isso, possam ser lidos por mais leitores.

\section{Considerações Finais}

Diante do exposto, observou-se que diversas áreas do Brasil já foram amostradas em relação a estudos com Briófitas em Unidades de Conservação. No entanto, algumas lacunas ainda são reconhecidas em regiões que permanecem insuficientemente amostrados e que precisam de maiores esforços de coleta. Ademais, estudos desenvolvidos, especialmente em áreas naturais protegidas certamente produzirão novos registros, possivelmente novas espécies de musgos, hepáticas e antóceros para a Flora Brasileira.

Com base no que foi analisado, em relação ao dominínio fitogrográfico mais amostrado em relação às Briófitas em Unidades de Conservação tem-se a Mata Atlântica, que também é o domínio fitogeográfico que apresenta maior riqueza de briófitas, seguido pelo domínio Amazônico. Vale ressaltar que esses números refletem principalmente os trabalhos realizados nos estados onde importantes grupos de pesquisas com briófitas estão instalados há mais tempo. Como é o caso dos estados de São Paulo e Rio de Janeiro na região Sudeste, Pernambuco e Bahia no Nordeste, Amazonas e Pará ao Norte, e que abrangem seus respectivos Biomas.

Percebe-se também uma baixa representatividade dos trabalhos com briófitas em UCs na Caatinga e Cerrado. Evidenciando que, embora trabalhos com briófitas tenham sido publicados nos últimos anos, em regiões que contemplam estes 
domínios, pesquisas com briófitas em Unidades de Conservação ainda são pouco exploradas. Alia-se a isso a falta de especialistas trabalhando com briófitas em alguns estados do Brasil.

E por fim, ressalta-se a importância dos resultados desse trabalho, em relação a apontar as lacunas aqui mencionadas, no que diz respeito ao conhecimento sobre briófitas em UCs e ainda reforça a importância em formar novos recursos humanos em algumas regiões do Brasil pouco representada nas publicações com esses vegetais, uma vez que o número de pessoas no país capazes de identificar briófitas ainda é extremamente limitado e representa o maior impedimento ao conhecimento da diversidade do grupo. E com isso, chamar atenção da necessidade em catalogar a brioflora presente nessas áreas prioritárias para a conservação da biodiversidade, e assim, reforçar a importância da manutenção de áreas protegidas.

\section{Referências}

Barros, F. S. M., Siqueira, M. F., \& Costa, D. P. (2012). Modeling the potential geographic distribution of five species of Metzgeria Raddi in Brazil, aiming at their conservation. The Bryologist, 15 (2), 341-349. http://dx.doi.org/10.1639/0007-2745-115.2.341

Camara, P. E. A. S., \& Van Rooy, J. (2014). A new species of Sematophyllum (Sematophyllaceae) from South Africa and a key to the southern African species of the genus. Bryologist, 117 (3), 297-300. 10.1639 / 0007-2745-117.3.297

Capes (2020). Potal de Periódicos da Capes. https://www.periodicos.capes.gov.br/

Costa, F. B., Silva, E. O., \& Conceição, G. M. (2015). Hepáticas (Marchantiophyta) e musgos (Bryophyta) da Área de Proteção Ambiental do Buriti do Meio, município de Caxias, Maranhão, Brasil, Scientia Plena 11 (1).

Costa, D. P., \& Peralta, D. F. (2015). Bryophytes diversity in Brazil. Rodriguésia, 66 (4), 1063-1071. 2015. 10.1590/2175-7860201566409

Darosi, G. C. M., \& Anderle, D. F. (2014). Mapeamento da Produção Científica Internacional sobre Inteligência de Estado. Espacios. 35 (5). http://www.revistaespacios.com/a14v35n05/14350405.html

Deheuvels., Rousseau, G. X., Quiroga, G. S., Franco, M. D., Cerda, R., Mendoza, S. J. V., Somarriba, E. (2014). Biodiversity is affected by changes in management intensity of cocoa-based agroforests. Agroforestry Systems, 8 (3), 1081-1099. https://doi.org/10.1007/s10457-014-9710-9 2014.

Flora do Brasil (2020) em construção. Jardim Botânico do Rio de Janeiro. <http://floradobrasil.jbrj.gov.br/reflora/floradobrasil/FB128472

Frahm, J. P. (2003). Diversity of bryophyte species on the tropics. In J.-P. Frahm (Ed.), Manual of Tropical Bryology, pp. 13-22. Tropical Bryology.

Glime, J. M. (2017). Introduction. Chapt. 1. In: Glime, J. M. Bryophyte Ecology. Volume 1. Physiological Ecology. Ebook sponsored by Michigan Technological University and the International Association of Bryologists. https://digitalcommons.mtu.edu/cgi/viewcontent.cgi?referer=\&httpsredi $\mathrm{r}=1$ \&article=1000\&context=bryo-ecol-subchapters.

Goffinet, B., Buck, W. R., \& Shaw, A. J. (2009). Morphology and Classification of the Bryophyta. In: Goffinet, B. \& Shaw, A.J. (eds.). Bryophyte Biology. New York: Cambridge University Press, p. 55-138.

Gradstein, S. R., Churchill, S. P., \& Salazar-Allen, N. (2001). Guide to the bryophytes of Tropical America. Memoirs of the New York Botanical Garden 86: $1-57$.

Kaffer, M. I., Koch, N. M., Martins, S. M. D., \& Vargas, V. M. F. (2016). Lichen community versus host tree bark texture in an urban environment in southern Brazil. Iheringia Serie Botanica, 71 (1), 49-54

Mägdefrau, K. (1982). Life-forms of bryophytes. In Bryophyte Ecology (A.J.E. Smith, ed.). Chapman and Hall. Cambridge University Press, p. 45-58.

Medeiros, R., Young; C. E. F., Pavese, H. B., \& Araújo, F. F. S. (2011). Contribuição das unidades de conservação brasileiras para a economia nacional. Brasília: UNEP-WCMC, 44p.

MMA-Ministério do Meio Ambiente. Áreas Protegidas. http://www.mma.gov.br/areas-protegidas/unidades-de-conservacao.html.

Milani, L. R., Prezoto, F., Clemente, M.A., Gomes, P. P., \& Souza, M. M. (2020). Nesting Behaviour of a Neotropical Social Wasp Mischocyttarus saussurei Zikán, 1949 (Hymenoptera, Vespidae). Sociobiology 67 (1), 121-125. 10.13102/sociobiology.v67i1.4842

Oliveira, R. R.; Oliveira, H. C.; Peralta, D. F.; \& Conceição, G. M. (2018). Acrocarpic mosses (Bryophyta) of Chapada das Mesas National Park, Maranhão, Brazil. Check List 14(6): 967-975. 10.15560/14.6.967

Pôrto, K. C., Silva, I., Reis, L., \& Maciel-Silva, A. S. (2016). Sex ratios and sporophyte production in the moss Bryum argenteum Hedw. on a rock outcrop, north-eastern Brazil. Journal of Bryology, 38 (4), 194-198.

Rylands, A. B., \& Brandon, K. (2005). Brazilian protected areas. Conservation Biology, 119 (3), 612-618.

Reis, L. C. (2018). Briófitas epífitas em agroflorestas de cacau na Floresta Atlântica. (Tese Programa de Pós-Graduação em Biologia Vegetal). Universidade Federal de Pernambuco. Recife/PE. 
Research, Society and Development, v. 10, n. 8, e4610816940, 2021

(CC BY 4.0) | ISSN 2525-3409 | DOI: http://dx.doi.org/10.33448/rsd-v10i8.16940

Santos, R. C. P., \& Lisboa, R. C. L. (2003). Musgos (Bryophyta) do Nordeste paraense Brasil-1 Zona Bragantina, Microrregião do Salgado e Município de Viseu. Acta Amazonica 33 (3), 415-422.

Silva, M. P. P., Kamino, L. H. Y., \& Porto, K. C. (2014). Is the current network system of protected areas in the Atlantic Forest effective in conserving key species of bryophytes? Tropical Conservation Science, 7 (1), 61-74.

Shepherd, G. J. (2003). Avaliação do Estado do Conhecimento da Diversidade Biológica do Brasil /Plantas Terrestres, Unicamp.

WWF-Brasil (2010). Consulta pública e Unidade de Conservação. Fundação Florestal do Estado de São Paulo, Instituto Florestal, Secretaria do Meio Ambiente do Estado de São Paulo. 